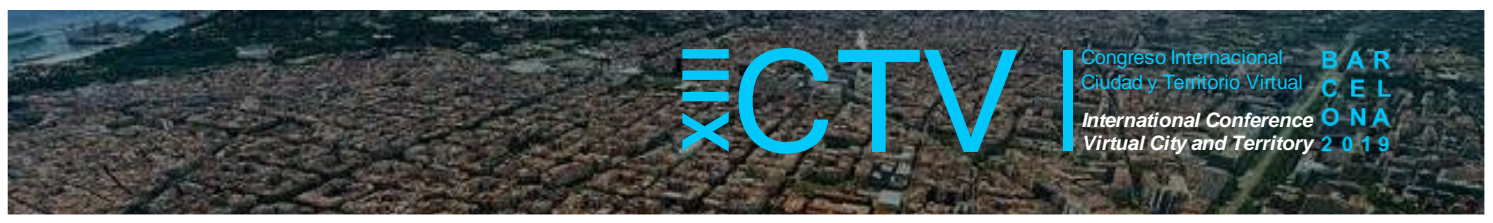

\title{
EL ESPACIO PORTUARIO DE A CORUÑA COMO UN CAMPUS TIC SOSTENIBLE PARA LA UDC
}

\author{
López-González, Cándido ${ }^{1 *}$; Carreiro-Otero, María ${ }^{2}$ y López-Carreiro, Iria ${ }^{3}$
}

Remisión inicial: 2019-06-06; Remisión definitiva: 2019-08-28; Publicación: 2019-12-21

Citación: López-González, C. et al. (2019). El espacio portuario de A Coruña como un campus TIC sostenible para la UDC. En XIII CTV 2019 Proceedings: XIII International Conference on Virtual City and Territory: "Challenges and paradigms of the contemporary city": UPC, Barcelona, October 2-4, 2019. Barcelona: CPSV, 2019, p. 8511. E-ISSN 2604-6512. DOI http://dx.doi.org/10.5821/ctv.8511

Resumen

Objetivo: El texto pretende determinar la idoneidad de los muelles de Batería y Calvo Sotelo en el área portuaria de A Coruña para destinarse a un "novedoso" uso, el de campus sostenible de las Tecnologías de la Información y la Comunicación -TIC- de la Universidade da Coruña -UDC-. Una actividad inédita en las actuaciones portuarias más recientes que conlleva fijar las cualidades del lugar.

Metodología: El método a utilizar se desarrolla a través de cuatro pasos. En el primero, se enuncia el prototipo de urbanización mayoritariamente empleado en las operaciones de remodelación portuaria. En el segundo, se plantea el puerto de A Coruña como caso de estudio; en concreto, se describen y analizan en las cartografías históricas y recientes los muelles de Batería y Calvo Sotelo y su entorno. En el tercer momento, se recogen en los archivos de la institución universitaria los datos que permiten reconocer el modelo de campus que la UDC desarrolla en el marco urbano. Y, por último, atendiendo a los criterios establecidos en diferentes estudios y guías de sostenibilidad para los campus universitarios, se determina una serie de dimensiones, asociándolas de modo biunívoco con las características propias del lugar. En consecuencia, este procedimiento permite identificar las capacidades del ámbito portuario estudiado para implantar en él los usos universitarios.

Desarrollo: Durante la última década del siglo XX, se conforma un paseo litoral que recorre la casi totalidad de la costa del T. M. -Término Municipal- de A Coruña, con la excepción de las áreas correspondientes al Puerto de la ciudad. En este momento, el traslado de diferentes actividades portuarias desde la dársena interior al puerto exterior de Langosteira, ubicado en el limítrofe T. M. de Arteixo, libera dos ámbitos portuarios, los muelles de Batería y Calvo Sotelo, con una superficie terrestre aproximada de 6,40 hectáreas. Surge así la oportunidad de concluir el paseo litoral, pero, sobre todo, la de analizar el destino del área portuaria como campus universitario de investigación vinculado a las TIC. Un uso compatible con las actividades soportadas por el espacio urbano central adyacente, y complementario tanto al tradicional uso pesquero, que se desenvuelve en los muelles de Linares Rivas y A Palloza, como al turístico, más moderno, que tiene lugar en el muelle de Trasatlánticos.

Para evaluar la incorporación de este nuevo modelo, se estudian los antecedentes de remociones portuarias, tanto internacionales como nacionales. A continuación, se realiza una aproximación histórica al lugar y una contextualización de la universidad en el entorno urbano de A Coruña. Finalmente, se incorpora la sostenibilidad como elemento clave para el desarrollo equilibrado del área portuaria, relacionando sus dimensiones con parámetros vinculados al puerto como enclave territorial y físico: dimensión histórica, con identidad; dimensión cultural, con reconocibilidad y complementariedad; dimensión ambiental, con accesibilidad y movilidad. Explorándose también, la dimensión social de la universidad que se concreta en la llamada tercera misión, la transferencia a la sociedad. Transferencia que reside no solo en las aulas y laboratorios, sino en su capacidad de ejemplificarse como referente de sostenibilidad, sea en su inserción en el espacio urbano, sea en su compromiso con la movilidad y la accesibilidad.

Conclusiones: El estudio establece una serie de cualidades propias del ámbito portuario que confirma el lugar de los muelles de Batería y Calvo Sotelo como un potencial polo tecnológico sostenible. La primera, la identidad singular que le proporciona la sedimentación de las diferentes capas históricas. La segunda, la imagen reconocible que le otorga el valor patrimonial de los diversos volúmenes edificados. La tercera, la complementariedad que le ofrece la diversidad de usos existentes en los espacios portuarios adyacentes. La cuarta, la localización central y en el plano bajo de la ciudad tanto le confiere una notable accesibilidad peatonal al mar como propicia la utilización de medios de transporte sostenibles. Así mismo, la transferencia social y económica fortalece la vinculación entre puerto y universidad, en una simbiosis que enfatiza el carácter urbano del puerto, pero también refuerza la presencia de la universidad en la ciudad.

${ }^{1}$ GAUS research group, Escuela Técnica Superior de Arquitectura, Universidade da Coruña y https://orcid.org/00000003-3388-6859; ${ }^{2}$ GAUS research group, Escuela Técnica Superior de Arquitectura, Universidade da Coruña, https://orcid.org/0000-0001-6351-7902 y ${ }^{3}$ TRANSyT, Centro de Investigación del Transporte, Universidad Politécnica de Madrid. * Correo de contacto: candido.lopez@udc.es 


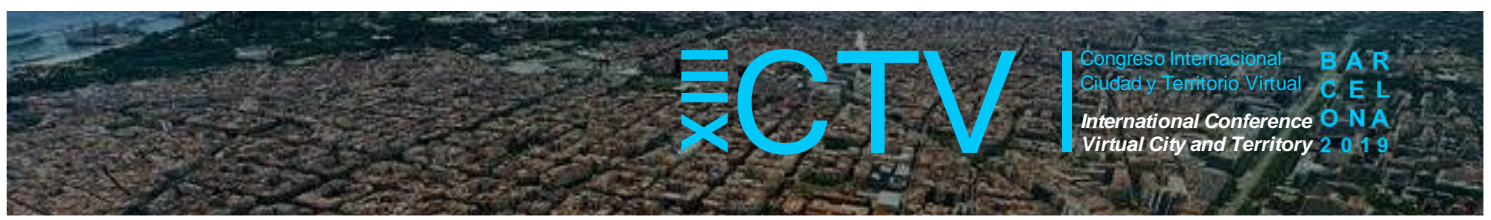

Una reflexión dirigida a las administraciones públicas implicadas en el tema: Autoridad Portuaria de A Coruña, Ayuntamiento de A Coruña, Ministerio de Fomento y UDC.

\section{Abstract}

Objective: The paper aims to illustrate the suitability of the quays of Batería and Calvo Sotelo for implementing an "innovative" use in the Port of A Coruña: the sustainable Campus of Information and Communication Technologies (ICTs) of the Universidade da Coruña (UDC). This is an unprecedented activity in the most recent port transformations that implies setting the qualities of the place.

Methodology: The methodological approach comprises four steps. First, the paper analyses the urbanisation models mostly applied in the regeneration strategies of port areas. Second, it defines the case study of A Coruña. Specifically, the research analyses the quays of Batería and Calvo Sotelo, and their evolution over time through the comparison of historical and present cartographies. Third, the study reviews the University archives for identifying the campus model that the UDC proposes in the urban framework. Finally, the research determines a set of sustainability dimensions and associates them with specific parameters of the port area. These dimensions are established in accordance with diverse studies and guides on sustainable university campuses. In summary, the methodology allows us to identify the potential of port areas for hosting university uses.

Discussion: In the last decade of the 20th century, almost the entire coastline of the Municipal Term of A Coruña is regenerated, with the exception of those areas corresponding to the Port of the city. In that moment, different port activities are transferred from the inner harbour to the outer port area of Punta Langosteira, in the municipality of Arteixo. As a consequence, two port areas of about 6.40 hectares -the quays of Batería and Calvo Sotelo- are released. This relocation allows to complete the city waterfront. But moreover, it provides an opportunity to analyse the suitability of the Port for hosting a University research campus linked to ICT. A complementary use with the ones developed in the urban area, and also with the fishing and tourist activities that respectively take place in the quays of Linares Rivas and A Palloza, and of Trasatlánticos.

With the objective to assess the implementation of this new model, the research analyses a series of existing projects focused on port regeneration, both at national and international levels. Then, it develops a historical approach to the place and contextualises the University in the urban framework of A Coruña. Finally, the notion of sustainability is incorporated as a key element for a balanced development of the port; and its dimensions are connected with specific parameters of the maritime area. The historical dimension is associated with the identity; the cultural dimension, with the recognisability and the complementarity; and the environmental dimension, with the accessibility and the urban mobility. The study also explores the social dimension of the University, which is reflected in the so-called third mission: the transfer (of knowledge) to society. This transfer resides not just in the teaching and research activities that characterise the academic institution, but also in its ability to become a reference of sustainability.

Conclusions: The study establishes a series of qualities that confirm the potential of the quays of Batería and Calvo Sotelo as a sustainable technological pole. The first quality refers to the unique identity of the area, provided by the sedimentation of the different historical layers. The second quality -the recognisable nature of the place- appreciates the heritage value of the various buildings located in the port. The third quality alludes to the complementarity of the diverse existing uses in the adjacent maritime spaces. Finally, the fourth quality addresses the location of the port area, which promotes pedestrian accessibility and the use of sustainable mobility options. Moreover, social and economic transfer reinforces the connection between the port and the University, in a symbiosis that emphasizes the urban character of the port, and also encourages the presence of the University in the city.

Our reflection is addressed to the public administrations that are involved in the subject: Autoridad Portuaria de A Coruña, Ayuntamiento de A Coruña, Ministerio de Fomento and UDC.

Palabras Clave: puerto; revitalización urbana; fachada marítima; campus universitario

Key words: port; urban revitalization; maritime facade; University campus

\section{Notas iniciales}

El concepto de sostenibilidad del Informe Brundtland (1987, 23), presente en las políticas medioambientales desde la última década del siglo XX, establece la condición de "satisfacer las necesidades del presente sin comprometer la capacidad de las futuras generaciones para satisfacer las propias". Un requisito que Navas $(2008,7)$ complementa, al manifestar que "tanto las organizaciones gubernamentales como las organizaciones empresariales tienen un alto grado de responsabilidad en la apuesta por el desarrollo sostenible". 


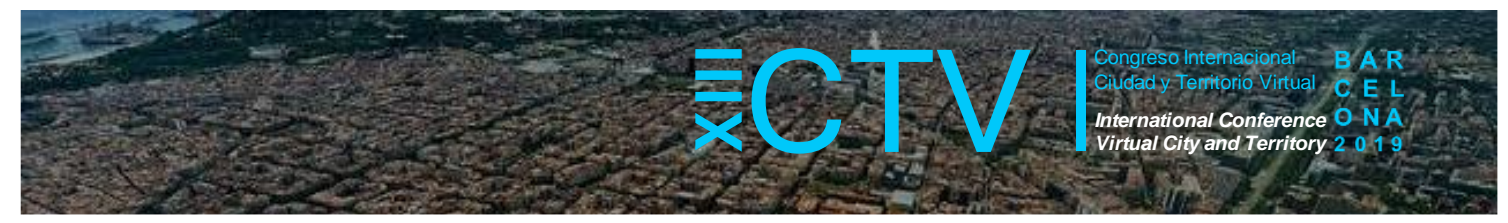

La necesidad de integrar la sostenibilidad en el sistema portuario resulta evidente. Por otro lado, el valor histórico, patrimonial y paisajístico, así como la importancia funcional y económica de las áreas portuarias, les confieren una notable potencialidad de desarrollo. Unos lugares que han establecido con la ciudad una relación de un continuo vaivén en distintas épocas (Hoyle, 1994 y 1997; Barragán, 1995; Navarro, 1998; Meyer 1999). Hoy, la transformación del espacio portuario en las ciudades marítimas supone una de las mayores oportunidades urbanas. Esta experiencia se inicia en Norteamérica ${ }^{2}$ en los años 60 y 70 del siglo XX, trasladándose a Europa a partir de los años 80 . Primero a Gran Bretaña ${ }^{3}$ y posteriormente al resto de países europeos. Sin embargo, las condiciones geográficas, económicas, y culturales de cada ciudad impiden extraer conclusiones generales que sirvan de modelo (Casariego et al., 1999). En España, en las operaciones de remodelación portuaria priman los contenidos comerciales frente a otros usos (Pozueta, 1996). Remodelaciones integrales como las de Santander y Gijón, o parciales como el Moll de la Fusta de Barcelona. A ellas, se les suman Málaga, Algeciras, Valencia, Vigo, Almería, o A Coruña en la primera década del siglo XXI. Algunos de estos casos han fracasado por fijar un modelo descontextualizado (Alemany, 2006).

Durante el último decenio del siglo XX, el Puerto de A Coruña, el Ayuntamiento de A Coruña, y la Demarcación de Costas del Estado y la Dirección General de Carreteras del entonces Ministerio de Obras, Transporte y Medio Ambiente realizaron un conjunto de actuaciones en $\mathrm{A}$ Coruña que conformaron el paseo litoral que recorre la costa, con la excepción de las áreas correspondientes al Puerto de la ciudad y a los extremos más periféricos, el lugar de As Xubias y los ámbitos de O Portiño y Bens. En este momento, el traslado de diversas actividades de cabotaje desde la zona portuaria de la bahía interior al "puerto exterior" de Punta Langosteira, ubicado en el T. M. de Arteixo, posibilita concluir uno de estos tramos no realizados, el correspondiente a los muelles de Batería y Calvo Sotelo.

El convenio suscrito, en 2004, entre el Ayuntamiento de A Coruña y el Ministerio de Fomento recogía el compromiso de reordenar urbanísticamente estos terrenos desafectados (Fig. 1) en el plazo máximo de cuatro años. Así mismo, incorporaba la necesidad de costear las obras del recinto portuario exterior con la venta del suelo desocupado. Para ello, en los terrenos de los muelles de Batería y Calvo Sotelo se preveían usos comerciales, terciarios y de ocio. Por otra parte, el Plan General de Ordenación Municipal de 2009 le asignaba funciones comerciales y residenciales, combinadas con zonas verdes y de recreo. La privilegiada ubicación del ámbito ha provocado la confrontación entre la Autoridad Portuaria y el Gobierno Local. Este último convocó un concurso de ideas, en 2018, para integrar los espacios portuarios en la ciudad. A pesar del alcance del tema, la convocatoria suscitó escaso interés entre los colectivos profesionales. Prueba de ello es el exiguo número de proyectos que se presentaron al concurso. Frente a la idea que los concursantes tenían de la futura evolución del puerto, se encontraban las realidades y exigencias del momento, que no parecieron haber encontrado su lugar en la formulación de las necesidades. Y aunque el concurso se falló con la adjudicación de cinco premios, la sombra del programa condicionó en gran medida las respuestas de los proyectistas.

\footnotetext{
${ }^{2}$ Lugares como Baltimore, Boston, San Francisco, Nueva York, Seattle o Savannah ocupan el frente portuario con usos fundamentalmente de tipo terciario.

${ }^{3}$ Londres con la operación de los London Docklands iniciará estos procesos en 1981. Posteriormente, a menor escala, aparecen las intervenciones de Liverpool y Southampton.
} 


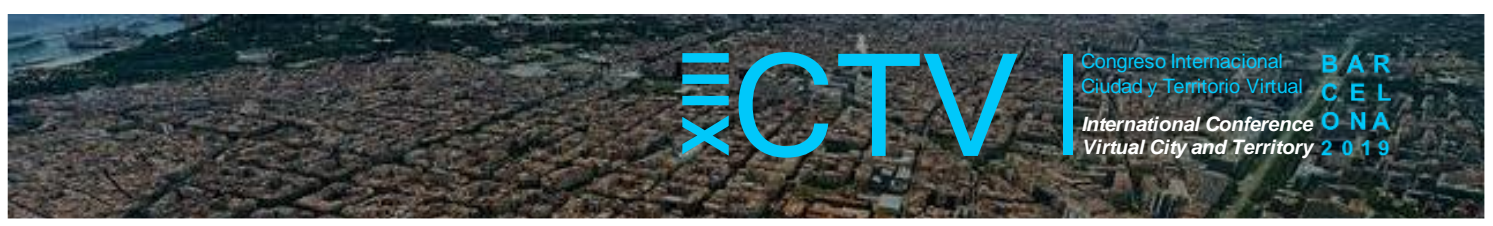

Frente a los modelos de urbanización que se proponían, cabe explorar una alternativa que implica a una institución educativa y no ha sido tenida en cuenta. Las autoridades académicas de la Universidade da Coruña -UDC- han manifestado públicamente, y en reiteradas ocasiones, la necesidad de crear un espacio especializado que complete su oferta formativa. No cabe duda que esta demanda real, introducir un campus con equipamientos universitarios públicos vinculados a las TIC, y por ende espacios públicos y zonas verdes, constituye un caso de estudio. En consecuencia, el texto pretende establecer las cualidades del ámbito de los muelles de Batería y Calvo Sotelo para destinarse a una actividad inédita en las actuaciones portuarias más recientes, el uso universitario. Este "novedoso" uso, el de campus sostenible TIC de la UDC, se propone como una opción compatible tanto con las actividades existentes en el área portuaria como con las soportadas por el espacio urbano central adyacente de A Coruña.

Figura 1. Muelles de Batería y Calvo Sotelo en el entorno urbano

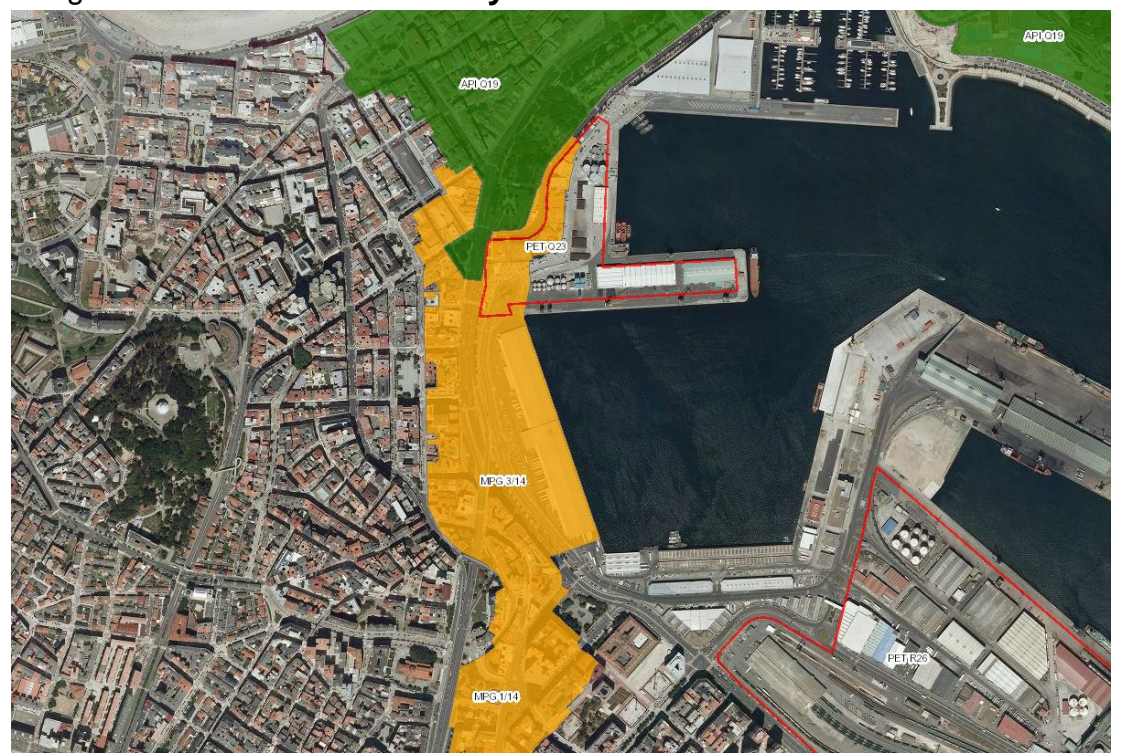

Fuente: Fuente: Ayuntamiento de A Coruña. Plan General de Ordenación Municipal 2009

\section{Metodología}

El método empleado comprende cuatro pasos. En un primer momento, enuncia el prototipo de urbanización mayoritariamente utilizado en las operaciones, tanto internacionales como nacionales, de remodelación portuaria. En segundo, se plantea el lugar de A Coruña como caso de estudio; en concreto, se analizan pormenorizadamente los muelles de Batería y Calvo Sotelo y su entorno en las cartografías históricas y recientes, describiendo tanto su evolución como las actividades y construcciones que actualmente acogen. En un tercer momento, se recogen en los archivos universitarios los datos que permiten reconocer el modelo de campus que la UDC desarrolla en el marco urbano. Y, por último, atendiendo a los criterios establecidos en diferentes estudios y guías de sostenibilidad para los campus universitarios, se determina una serie de dimensiones, asociándolas de modo biunívoco con las características propias del lugar. La dimensión histórica con la identidad; la cultural con la reconocibilidad y complementariedad; la ambiental con la accesibilidad y movilidad; y la social con los efectos simbióticos urbanos. Este método permite identificar las capacidades del ámbito portuario estudiado para implantar en él los usos universitarios. 


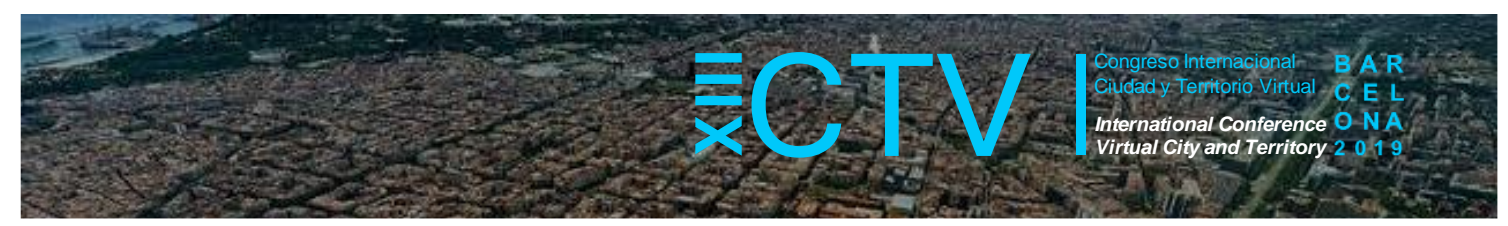

\section{Antecedentes}

\subsection{El enclave portuario}

En sus orígenes, en A Coruña puerto y ciudad aparecen como un todo, unidos (Acinas, 1998). La playa del Parrote, que inicialmente acoge a las embarcaciones que realizan tanto las faenas de pesca como los intercambios de mercancías, se extiende entre los siglos XIII al XVI hacia el interior de la bahía coruñesa ocupando los arenales protegidos del istmo. En él, el enclave portuario de nuestro interés se localiza en el punto central del arco formalizado entre las actuales Plazas de María Pita y de la Palloza. El lugar, que organiza la playa interior de la bahía en dos zonas, aparece en las primeras cartografías del siglo de la razón como un promontorio rocoso (Fig. 2). Un elemento natural que el ingeniero Director de Galicia del Cuerpo de Ingenieros Militares Francisco Montaigú de la Perille dibuja minuciosamente en la serie de proyectos que realiza entre 1723 y 1726 . En el último de los ideados en 1726, el lugar se formaliza geométricamente mediante un semibaluarte. Este elemento, que acoge una construcción defensiva, es el germen del hoy denominado Muelle de Batería (Soraluce, 1985). La propuesta aúna la defensa y crecimiento de la ciudad con el desarrollo del puerto. Sin embargo, los proyectos a lo largo del siglo XVIII se decantarían por atender a las transformaciones de uno $u$ otro ámbito 4 por separado.

La segregación del espacio portuario del urbano se formaliza a mediados del siglo XIX mediante un recinto administrativo con sus propias reglas. A pesar de ello, en 1860, el puerto se encuentra en un estado de abandono general. "Las mercancías han de ser transportadas por carros y hombres a lo largo del arenal" en un ambiente poco decoroso para una capital y un puerto de la importancia de La Coruña (Uribe, 1866; Vila, 1909, 16). En los dos proyectos que se ofrecen como solución, el lugar denominado Batería de Salvas o el Malvecín (Reiriz, 1999) se considera una referencia relevante. Si en el primero de ellos, redactado en 1857 por el ingeniero José Bellón, es el punto inicial que aloja el edificio del Parque de Ingenieros Militares; en el segundo, ideado en 1860 por el también ingeniero Celedonio de Uribe, es el punto final de un trazado con inicio en el Baluarte del Parrote. Una Batería de Salvas que consolida su papel central en los tres anteproyectos de ampliación global del puerto realizados en 1884, 1887 y 1891, por Eduardo Vila, ingeniero Director del Puerto. En todos ellos aparecen dos muelles que configuran la Dársena de La Palloza, un recinto entre el hoy Muelle de Calvo Sotelo y el Muelle del Este. Pero mientras este último se construye en la primera década del siglo XX, el primero, también denominado "de Transatlánticos", ha de esperar a 1923 para ser proyectado por el ingeniero Emilio Pan de Soraluce, a 1936 para verse acabado (Vigo Trasancos, 2014), y a la década de los 40 para recibir la línea ferroviaria y las primeras construcciones.

Una creciente demanda de espacio para alojar diversas actividades desemboca, en 1946, en el Proyecto General de las Obras y Mejoras del Puerto. Entre otras actuaciones, se propone la construcción del Dique de Abrigo. ${ }^{5}$ Con ello, se genera una especialización zonal del ámbito portuario, cuyas actividades ${ }^{6}$ resultan "incompatibles" con el libre acceso de los ciudadanos. La

\footnotetext{
${ }^{4}$ Reflejo de ello son los proyectos de Miguel Marín en 1755, el formulado por comisión de Miguel de la Puente en 1788, o los realizados por Giannini en 1789 y 1792, que atienden a la infraestructura portuaria en exclusiva.

${ }^{5}$ La obra dura prácticamente dos décadas, comienza a finales de 1948 terminándose en septiembre de 1965.

${ }^{6}$ Entre otras, se encuentran el control y la fiscalidad de los productos, la guarda y custodia de las mercancías, o la seguridad tanto de los estibadores como de las personas que circulan por su ámbito.
} 


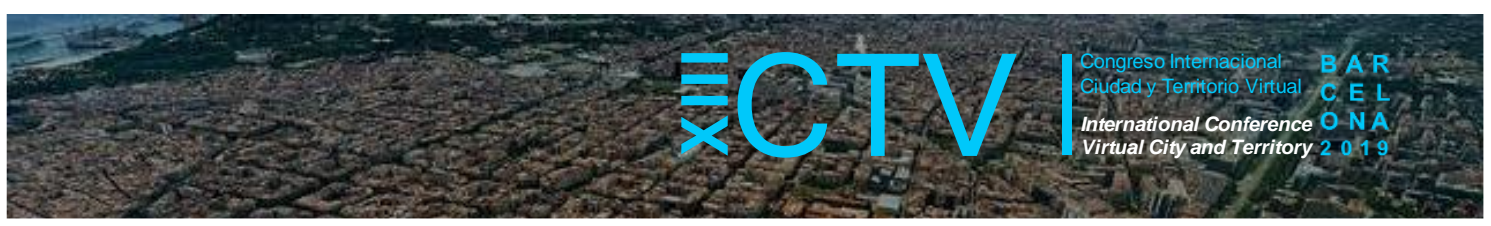

disociación urbe-puerto se refuerza al producirse el cierre y vallado del perímetro portuario. A partir de estos años, el Muelle de Calvo Sotelo se destina a la carga y descarga de graneles sólidos mientras el de Batería se reserva para el atraque de buques de uso turístico. Aunque en las siguientes décadas, el incremento de calado de esas naves, cada vez de mayor tamaño, dificulta su atraque. La consecuencia de la progresiva decadencia del uso turístico deriva en que este muelle también se ocupe con mercancías diversas.

Figura 2. Fotomontaje: Área portuaria de A Coruña (Planes Directores de Ordenación) sobre el plano realizado por Miguel Marín de 1755

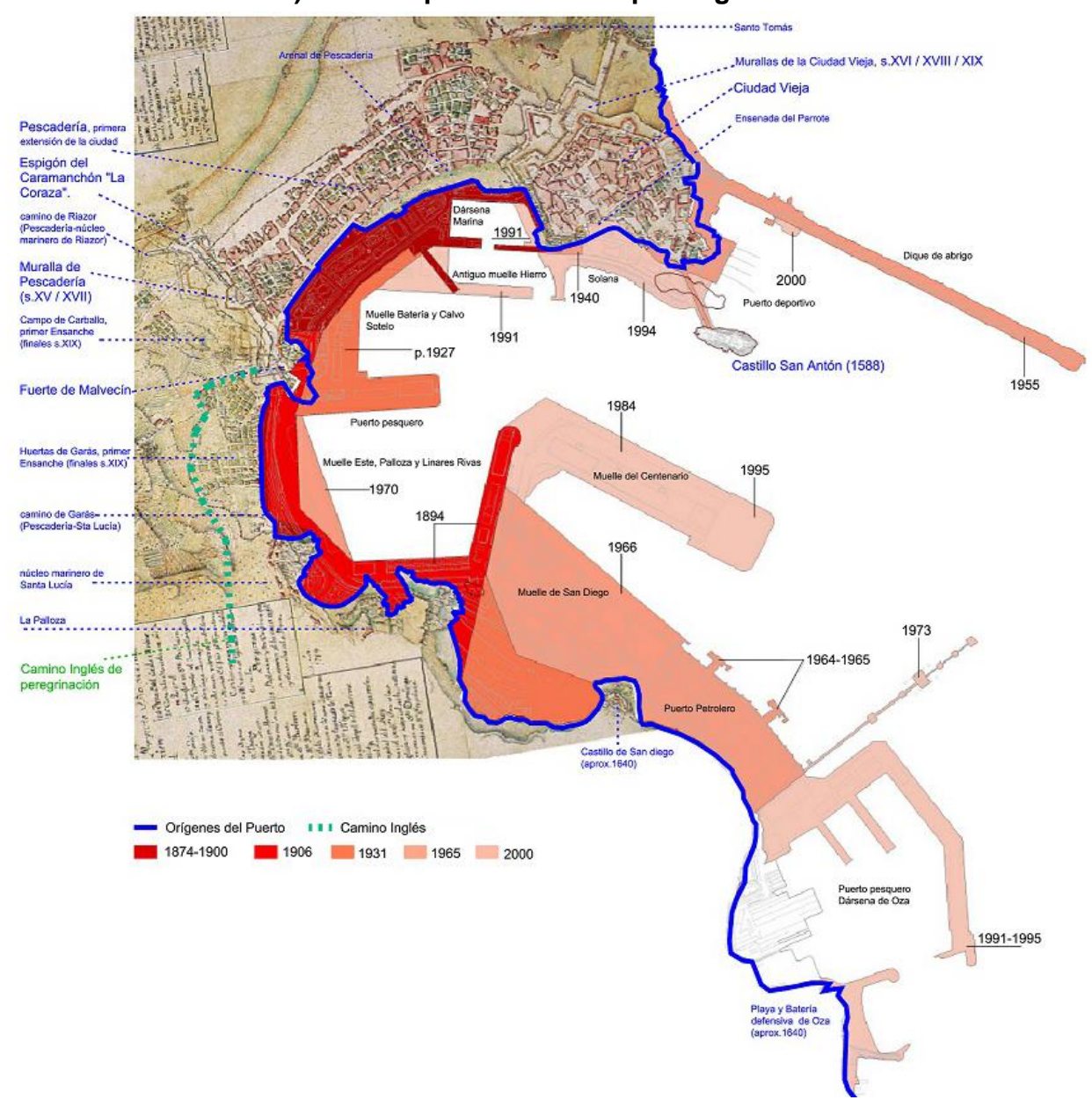

Fuente: Cotoya Díaz, Vanesa. El pasado del puerto de A Coruña

En la actualidad, ambos muelles, Batería y Calvo Sotelo (Fig. 3), con una superficie terrestre de 6,40 hectáreas, se organizan mediante una serie de áreas de depósito de mercancías y sus consiguientes espacios de circulación (Autoridad Portuaria A Coruña, 2018). El Muelle de Batería posee una superficie de 2,40 hectáreas aproximadamente. Lo ocupan una nave cerrada de almacenamiento de mercancía general, un área de almacenamiento al aire libre para productos siderúrgicos y, además, un conjunto cerrado de depósitos y silos, algunos de ellos con un aspecto de considerable deterioro. Todas ellas se sirven de unas zonas de vialidad que representan prácticamente el cincuenta por ciento de su superficie. El Muelle de Calvo Sotelo, con 4,00 hectáreas, completa el fragmento portuario. En su punta transversal aloja una construcción de escasa superficie y volumen, realizada en los años 50 , que contiene un 


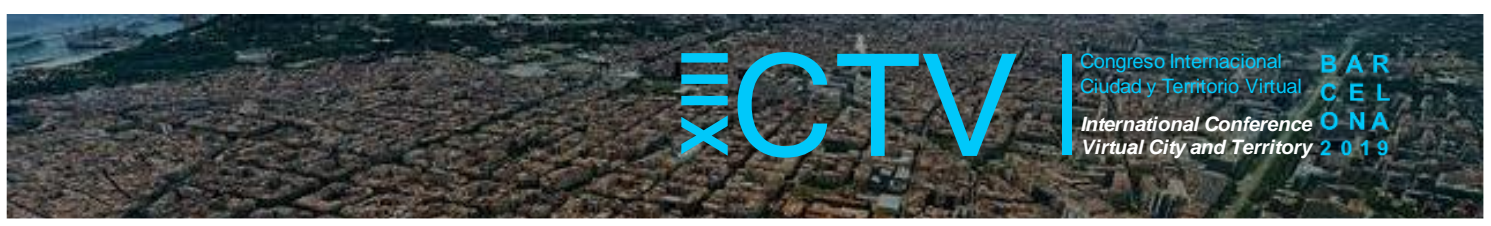

Mareógrafo. Este muelle dispone de dos naves cerradas, una para mercancía general y otra para mercancía general y frutas, ${ }^{7}$ junto con un área de almacenamiento al aire libre. Así mismo posee una serie de depósitos y silos. Unos para graneles sólidos, y otros para graneles líquidos. Entre ambos, un pequeño pabellón de oficinas de la Autoridad Portuaria presenta una descuidada imagen. También en este caso, los viales comprometen la mitad de su extensión.

Figura 3. Usos de la zona de servicio del puerto interior de A Coruña

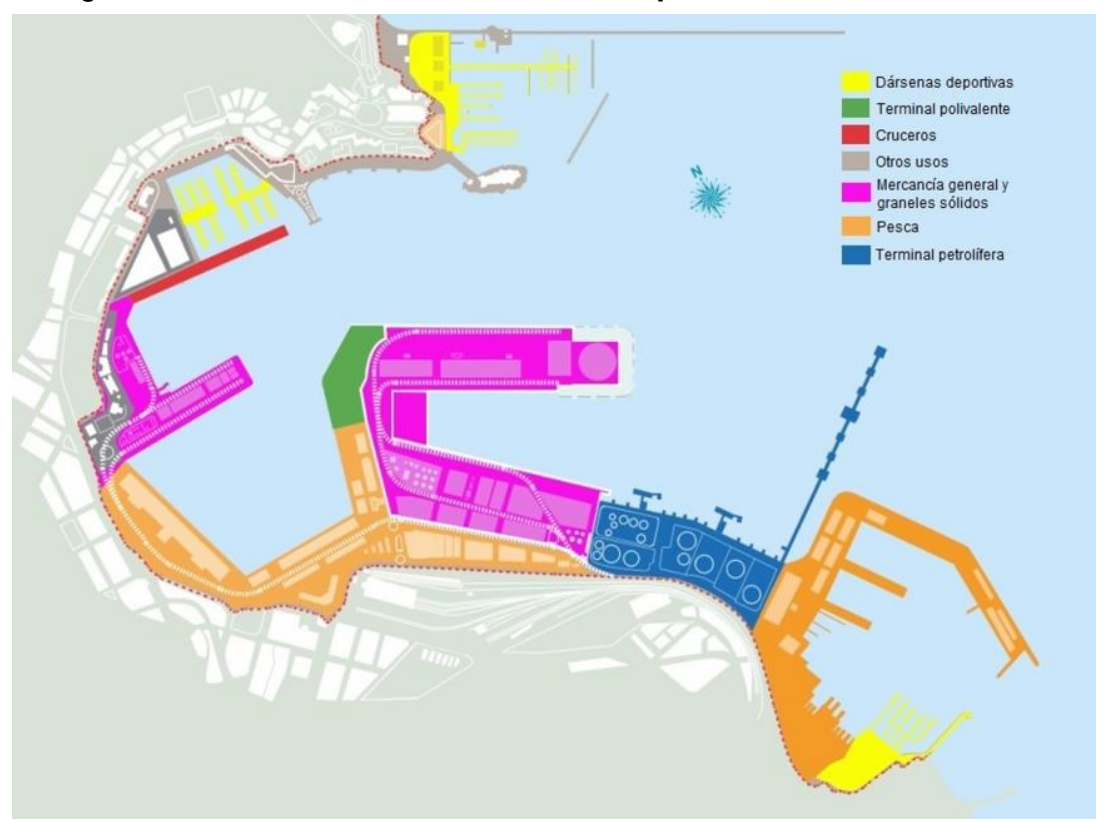

Fuente: Autoridad Portuaria de A Coruña. Memoria anual 2017: Puerto de A Coruña

A estos usos y construcciones se suman los existentes en tres ámbitos terrestres inmediatos: el muelle de Transatlánticos y la Dársena de la Marina, el muelle de Linares Rivas, o Garás, y el formalizado por una franja lineal ocupada por edificaciones singulares en contacto con los Jardines de Méndez Núñez o del Relleno.

El primero, ubicado al norte, organizado mediante un proceso de reestructuración que lideró la Autoridad Portuaria, consolidó la Dársena de la Marina como un recurso turístico. Una transformación llevada a cabo durante una década mediante una coordinada secuencia de operaciones. En 2005, se construyeron un palacio de congresos, Palexco, y el centro comercial adyacente. En 2011, se inauguró un muelle destinado a una nueva terminal de cruceros, con un calado de once metros. Y finalmente, en 2015, se creó un espacio para el peatón con una superficie de cinco hectáreas. Esta actuación implicó el soterramiento del tráfico, acompañado de la construcción de un aparcamiento subterráneo de alta capacidad.

El segundo, un área al sur que apuntaló su contenido como espacio de actividad pesquera. Así lo confirma la construcción de una gran lonja para la comercialización del pescado fresco en el Muelle de Linares Rivas para sustituir la Lonja del Gran Sol, ${ }^{8}$ ubicada en el muelle de La

${ }^{7}$ El almacén de frutas posee una capacidad de almacenamiento de $15.000 \mathrm{~m}^{3}$. Su propietario es la Autoridad Portuaria de A Coruña.

${ }^{8}$ Obra de los ingenieros Eduardo García de Dios y Félix Calderón Gaztelu realizada en 1960, no reunía las condiciones higiénico-sanitarias requeridas en la actualidad. Aunque en un evidente estado de descuido, ofrece una imagen reconocible con un característico perfil ondulado en las cubiertas resueltas con unas finas bóvedas de hormigón. 


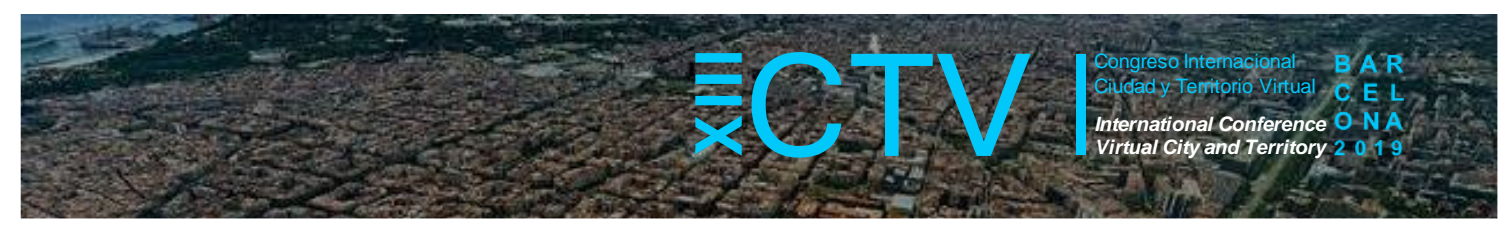

Palloza. El edificio, de 415 metros de longitud y $30.000 \mathrm{~m} 2$ de superficie útil, inaugurado en 2004 supone la adaptación a las exigencias comunitarias tanto de la conservación del pescado y marisco, como de la informatización de las subastas. Un complejo programa funcional da continuidad a las voces y contraseñas reglamentadas, provenientes del antiguo centro en el que se comercializaban las descargas del pescado fresco.

El tercero de ellos acogió una serie de equipamientos ${ }^{9}$ dispuestos en una sinuosa hilera, con sus fachadas principales a la Avenida do Porto. Los edificios de Portos de Galicia y de la Jefatura Superior de Policía presentan un considerable volumen construido, con cuatro y seis plantas respectivamente. Mientras, los inmuebles usados por la Dependencia Regional de Aduanas, el Ministerio de Defensa, o la Delegación de Gobierno se formalizan al modo de villas urbanas singulares, construidas a mediados del siglo XX con un lenguaje ecléctico. Por otra parte, en las edificaciones asociadas a la actividad portuaria se distinguen dos situaciones. Mientras que la pieza que aloja los transformadores eléctricos -en el extremo norte de la hilerase integra en el cierre portuario, la que alberga la cafetería-restaurante -en el extremo sur, actualmente en desuso- resulta de escaso interés, excepto por su moderado volumen. En cualquier caso, todos son inmuebles aislados que, en su discontinuidad, ofrecen la permeabilidad necesaria para que los peatones puedan disfrutar, directamente, del plano de la lámina del agua.

\subsection{Los campus de la Universidade de A Coruña en el marco urbano}

La organización territorial de la UDC parece aproximarse a un modelo "que no es jerárquico, a menudo basado en 'vocaciones' específicas de los centros particulares y basado también en intensas interacciones complementarias y sinérgicas" (Camagni, 1998: 37). Un paradigma de colaboración no subordinada que, implantado en redes regionales de pequeñas y medianas ciudades, implica la cooperación entre las universidades, el sector público y el sector productivo privado para coordinar espacios universitarios especializados.

Dentro del sistema universitario gallego, la UDC, creada en 1989, se estructura territorialmente en dos campus: el de A Coruña y el de Ferrol. En el primero, se reconocen seis ámbitos discontinuos, funcionalmente segregados. Los denominados como A Maestranza, Riazor, Elviña-Zapateira, Oza, Bastiagueiro, y Oleiros. El segundo, en la ciudad de Ferrol, se organiza en otros dos, también físicamente separados: Esteiro y Serantes. Todos ellos en posiciones urbanas diversas, con superficies y usos variados y una composición morfológica variopinta. ${ }^{10}$

En el caso de A Coruña, en cuanto a la posición en el territorio, los ámbitos de A Maestranza y Riazor se sitúan en el casco urbano consolidado; los de Elviña-Zapateira y Oza en áreas periféricas de expansión urbana; y los de Bastiagueiro y Oleiros en lugares de crecimiento extensivo, en el colindante término municipal de Oleiros. Considerando el parámetro de la superficie se observa una clara diferencia de magnitudes: desde el de mayor tamaño, Elviña-

\footnotetext{
9 Tanto diferentes organismos públicos -Portos de Galicia adscrito a la Consellería del Mar de la Xunta de Galicia, Jefatura Superior de Policía, Dependencia Regional de Aduanas e Impuestos Especiales de Galicia, Ministerio de Defensa y la Delegación de Gobierno- como dependencias auxiliares de las actividades portuarias -cafeteríarestaurante y local para transformadores eléctricos.

10 Una disposición que parece adecuarse al modelo surgido al calor del progreso económico y social ligado a la Revolución Industrial. En ese momento, el Saber se desagrega en multitud de nuevas ciencias que precisan espacios fragmentados y especializados. Ahí surge la cuestión que ha trascendido hasta nuestros días sobre la integración o segregación de esos espacios, tanto entre ellos como en su relación con la ciudad.
} 


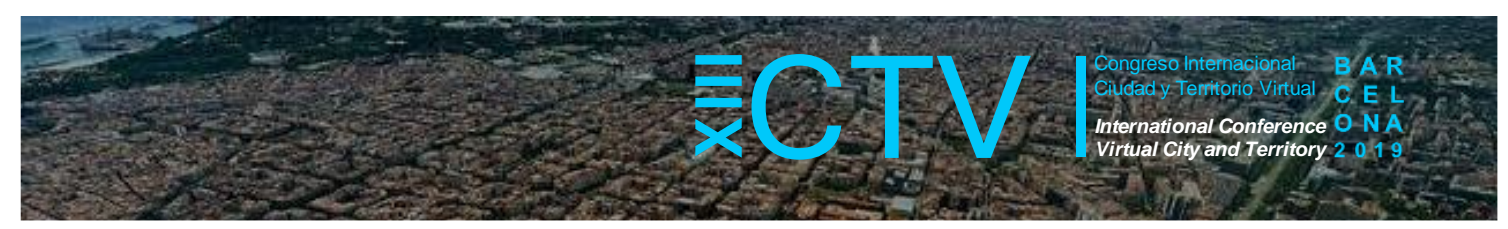

Zapateira con 51 has, hasta el menor, A Maestranza con 0,5 has, en el cual edificio y área coinciden. Una variedad dimensional que los singulariza. Si se atiende a la función de uso se constata la especialización en los cometidos que acogen. A Maestranza se identifica por los usos administrativos y representativos; Riazor, por el centro de enseñanza naútica, y los edificios culturales; Elviña-Zapateira por las escuelas técnicas, las facultades y los centros de investigación; Oza por los centros vinculados a las ciencias de la salud; Bastiagueiro por las instalaciones y edificios de las ciencias del deporte; y Oleiros, por una pieza de marcado carácter cultural. Unos elementos construidos que se decantan por la heterogeneidad en sus definiciones, conviviendo en una confusa amalgama. Además, constituyen un universo cerrado, sin que el estudiantado pueda desarrollar su ciclo vital diario en ellos: ninguno vive allí y todos se alejan una vez cumplidas sus obligaciones académicas.

En este contexto, la ubicación de un "nuevo" campus universitario, el de las TIC, ha de considerar la ciudad no solamente como una localización, sino también como un campo privilegiado de reflexión. El campus universitario es un organismo cuya implantación física, dotada de continuidad y flexibilidad, ha de acompañar al carácter dinámico de la institución. Más que un espacio especializado alojado en la ciudad, debe ser parte constituyente de la forma y el funcionamiento de la estructura urbana. Un recinto que, sustancialmente, se configure como sujeto de reordenación urbana. La ciudad es ella misma un recurso educativo importante y el campus puede potenciar su papel formativo y revalorizar sus múltiples recursos.

La revitalización del enclave portuario introduciendo el "nuevo" uso, no solo ha de entretenerse con cuestiones de configuración arquitectónica, sino que debe enfrentarse con complejos problemas de planeamiento, organización, gestión política, o competencias institucionales, junto con otros de tipo económico, financiero, jurídico y ecológico. En todos y cada uno de ellos se evidencia la necesidad de considerar la sostenibilidad como una dimensión transversal imprescindible para abordar la implantación universitaria.

\section{La sostenibilidad del uso universitario en los muelles de Batería y de Calvo Sotelo}

El reconocimiento de las dos situaciones, local una -el puerto como soporte físico -, territorial otra -los campus de las UDC- aporta verosimilitud a la opción de introducir en el enclave portuario un campus destinado a las TIC. Una alternativa que se aborda a través de un conjunto de parámetros vinculados a dicho enclave, conjuntamente con otro asociado a la universidad. En este caso, explorando la dimensión social de esta institución, que se concreta en la llamada tercera misión: la transferencia a la sociedad. Transferencia de conocimiento que reside, no solo en la producción docente e investigadora de aulas y laboratorios, sino en su capacidad de ejemplificarse como un referente de sostenibilidad ambiental, bien en su inserción en el espacio urbano, bien en su compromiso con la movilidad y la accesibilidad.

\subsection{La dimensión histórica: identidad}

"También los muelles pueden actuar más como costuras, al menos más de lo que suelen hacerlo hoy" (Jacobs, 2011, 303). En estos inicios del siglo XXI, permanece vigente la idea de incorporar los recintos portuarios como puntos estratégicos de sutura urbana, que la teórica del urbanismo y activista político-social Jane Jacobs expresó en los años 60 del siglo XX. El puerto de A Coruña ha sido históricamente un factor importante para el crecimiento económico y 


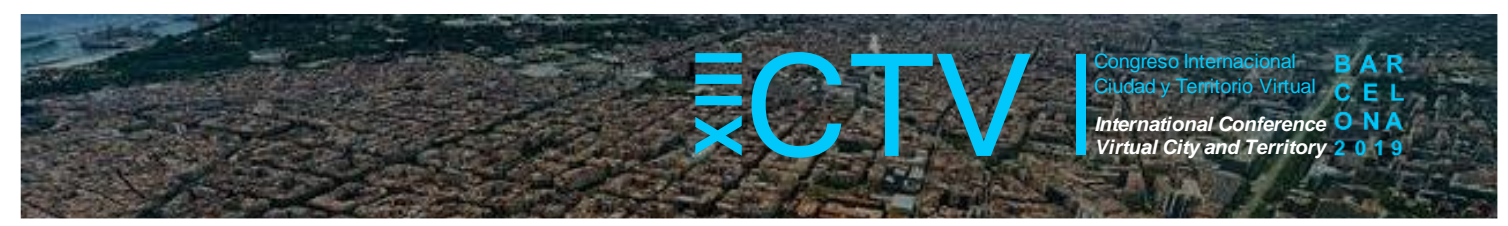

poblacional de la ciudad. Pero, al margen de factores económicos o técnicos, dos consideraciones socio-culturales reflejan su potencialidad (Grindlay, 2008). La primera, su emplazamiento central que posibilita un fácil acceso al mar de las personas. La segunda, su capacidad intrínseca para combinar nuevos equipamientos con la recuperación de construcciones histórico-patrimoniales para usos públicos.

Como recuerda Uriarte $(2007,57)$ "es preciso insistir en la permanencia de los testimonios más singulares y significativos en cada localidad del espíritu portuario que refuercen la idiosincrasia marítima de sus orillas". La conservación y transformación de las edificaciones de la época dorada del puerto constituyen una posibilidad única de integrar, constructiva y arquitectónicamente, pasado, presente y futuro. La clave, tal y como sugiere Alemany (2006, 25), pasa por "mantener el puerto en la ciudad, revitalizando las áreas abandonadas 0 subutilizadas, pero sin perder su carácter, para conservar la identidad, el patrimonio y aprovechar una oportunidad de desarrollo que sólo las ciudades portuarias permiten". Una determinación que la Carta internacional para el desarrollo sostenible de las ciudades portuarias ${ }^{11}$ también considera esencial. En su articulado, entre otros objetivos, se fija la necesidad de "respetar la identidad de los lugares", de "establecer nuevos principios de cohabitación ciudad/puerto" o de "manejar los distintos ritmos de la vida portuaria y de la vida urbana". A pesar de su actual desuso, las infraestructuras y edificaciones de los muelles son un testimonio relevante de la historia marítima y urbana de la ciudad.

Así pues, esta singularidad del lugar portuario -genius loci- se convierte en una oportunidad para dotar de nuevos usos a desocupadas estructuras industriales. La transformación de las instalaciones de los muelles de Batería y Calvo Sotelo para actividades universitarias TIC les permitiría recuperar su protagonismo urbano, proporcionándole al lugar una identidad colectiva. Este cambio se presenta como una oportunidad para poder afrontar algunas dificultades no resueltas de la organización de la ciudad: la reutilización de los barrios históricos, la reorganización del tráfico y del transporte local, la recolocación de algunos servicios o la reubicación de centros y funciones en el tejido urbano.

\subsection{La dimensión cultural: reconocibilidad y complementariedad}

El patrimonio industrial de singular valor tipológico representa un recurso para evitar el consumo de nuevo suelo. Un soporte para la reconfiguración de la urbe coruñesa, que además sirve de identificación de los ciudadanos con el puerto, memoria viva de un pasado común. Una reutilización vital en la relación puerto-ciudad, que Peña $(2012,16)$ define como "el hecho de dar nuevas funciones a espacios y edificios, cuando la finalidad y el uso que los hicieron nacer se vuelven obsoletos. Algunos están catalogados y otros no, pero sin estarlo, también forman parte del conjunto construido de la ciudad. Son edificios y espacios que en su día aportaron imagen a la ciudad y que en la actualidad aún están en buen estado; sin embargo, al caer en desuso, su actividad inicial debe sustituirse por otra".

Las administraciones públicas establecen una desigual protección a los diversos edificios de los muelles de Batería y de Calvo Sotelo. La autoridad portuaria y la institución municipal asignan un alto nivel de protección al Mareógrafo -construido en los años 50-, una pieza de

\footnotetext{
${ }^{11}$ Asociación Internacional de Ciudades y Puertos, AIVP: Carta para el desarrollo sostenible de las ciudades portuarias. Conferencia de Sydney (2006).
} 


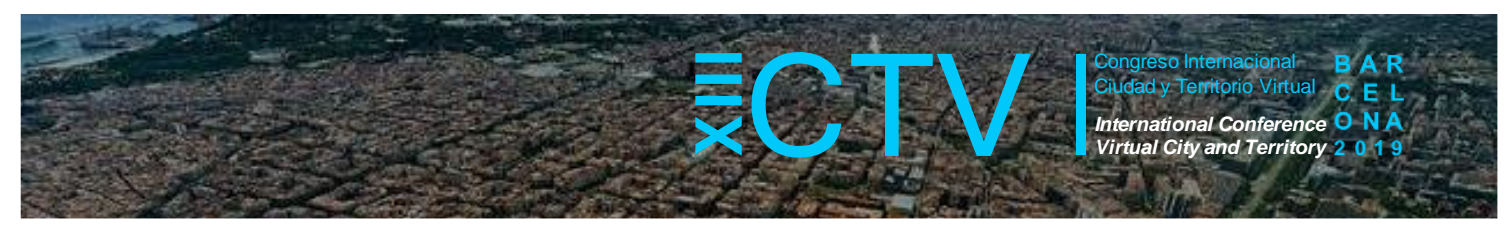

escala doméstica; y a los silos de Cementos del Cantábrico, un conjunto de volúmenes cilíndricos de gran pregnancia en el skyline portuario. Una actitud preservacionista que no se extiende al resto de las piezas construidas en los propios muelles y en su entorno más próximo. Si la autoridad portuaria contempla la protección de los edificios de Aduanas, Ministerio de Defensa ${ }^{12}$ y la Delegación de Gobierno, el planeamiento municipal únicamente la considera en el último de los edificios citados. Discrepancias que ponen en riesgo una intervención que revalorice su valor patrimonial.

La recuperación de construcciones históricas patrimoniales para usos públicos en la frontera marítimo-terrestre incorpora, en escasas ocasiones, la investigación y la tecnología (Boubacha et al. 1997) como una actividad, pese a ser una función compatible con otras, conocidas como actividades "blandas" (Fernández Pérez, 1999). Sean estas, terminales para tráfico de pasajeros, o de cruceros, dársenas deportivas, muelles para embarcaciones de los prácticos, remolcadores o avituallamiento, y usos pesqueros. Por otra parte, las medidas de naturaleza social que producen efectos favorables en áreas del puerto garantizando la accesibilidad de las personas al mar pasan, como expone Martín (2009), por fomentar paseos, plazas o balconadas al puerto, prácticas deportivas y recreativas, carril-bici, actividades de turismo, restauración o comercio, o por celebrar eventos especiales. Es evidente que una controlada actividad industrial y de servicios vinculada a los puertos, fija unas condiciones de convivencia y de contacto físico con las urbes, armonizando la doble vocación comercial y ciudadana de estos enclaves marítimos. Todas ellas son actividades complementarias que, introducidas equilibradamente, garantizan el adecuado uso del espacio portuario.

\subsection{La dimensión ambiental: accesibilidad y movilidad}

Conviene recordar las dos estrategias de intervención que propone Muñoz $(2006,42)$ contra la tendencia a la estandarización de los espacios portuarios. En primer lugar, la contextualización, que "configura el proceso de transformación a partir de las características físicas, pero sobre todo sociales y culturales del contexto local". En segundo, la lentitud como mecanismo que evita la rápida rotación de actividades y usuarios, creando espacios de estancia con diferentes posibilidades de utilización.

Las actuaciones en el área del puerto en desuso han de basarse en el principio de la ciudad compacta, dura y ecológica. En ella, las nuevas tecnologías han de considerar el modelado de espacios de alta calidad ambiental, interconectando corredores naturales y actividades innovadoras. Tecnologías que, mediante el big data, la inteligencia artificial, el internet de las cosas y la realidad aumentada, gestionan una exhaustiva información sobre accesibilidad y movilidad. Entre A Coruña y su poblado entorno metropolitano se genera un denso tráfico de carácter pendular. Un tránsito que se dificulta por la geografía peninsular de la urbe, la red de trazados del autobús -carente de un diseño integral desde hace treinta años-, y la falta del carril bus. Además, el modelo de aparcamiento de rotación en superficie, acompañado de un gran aparcamiento subterráneo en su punto central, genera un "excesivo tráfico de agitación" en la Avenida do Porto, el eje costero de penetración al centro histórico. Al igual que Samperi (1986) reconoce en un caso similar, el área de los muelles de Batería y Calvo Sotelo se encuentra

12 El inmueble, que se construyó en los años 50, está ocupado desde 2017 por la Oficina de Gobierno Local del Ministerio de Defensa. Con anterioridad, alojó la Comandancia Naval de Marina. En ella, se otorgaban al tráfico naval militar los permisos necesarios tanto para el atraque como aquellos relacionados con la logística de las embarcaciones. 


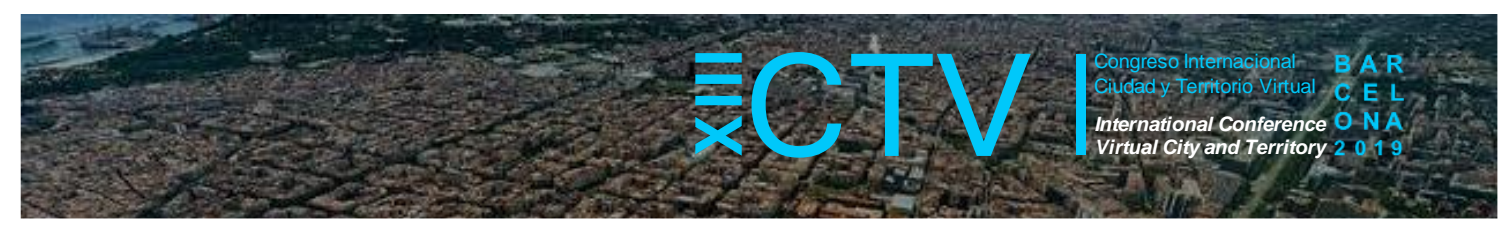

delimitada por una vía de alta capacidad rodada, que la separa del entorno urbano próximo. Si se pretende potenciar la accesibilidad, conviene definir el rol que juega este eje. ¿Es plausible continuar el soterramiento o semisoterramiento de otra parte? ¿Cómo repercutiría en la relación de la ciudad con el área portuaria? ¿Cuánto mejoraría la calidad de estos espacios representativos de la propia ciudad y cuál sería su repercusión en la vida cotidiana del ciudadano?

La accesibilidad al puerto, que genera la interacción de la ciudad y la ciudadanía con el mar y su memoria portuaria se considera una prioridad. Posiblemente se trate de anteponer el peatón frente al vehículo, el transporte público frente al privado, o de establecer otras formas de movilidad como la bicicleta, el patinete o la moto eléctrica, los coches autónomos, los robots mensajeros, o los drones. Una movilidad sostenible que garantice el protagonismo del peatón con espacios específicos, mejorando la comodidad de las vías peatonales y reduciendo los riesgos de atropello. Todas estas alternativas incrementan la calidad ambiental mediante el aumento del confort climático, la absorción de ruidos o la conformación de lugares de esparcimiento.

\subsection{La dimensión social: la 3ª misión de la universidad}

La recuperación arquitectónica de los edificios portuarios implica la consideración de tres líneas argumentales, la funcional, la cultural y la simbólica. La funcional se resuelve adecuando los volúmenes sólidos y el vacío entre ellos a las necesidades precisas, y previendo la flexibilidad. La cultural obliga a la adaptación al medio físico con elementos de intermediación entre el modelo organizativo de la universidad y el ámbito urbano. Y la simbólica, alusiva al carácter emblemático de la arquitectura, exige cuidar la imagen de la universidad.

En el proceso de reutilización urbanística, y de innovación en las políticas urbanas, es preciso considerar algunos aspectos relevantes. En primer lugar, atender a la peculiaridad del espacio universitario, que con frecuencia únicamente se expresa en términos de superficie utilizable. En segundo, sopesar las dificultades de transformación de los edificios existentes debido a sus características tipológicas. En tercero, valorar la ubicación de estos espacios, ante la conveniencia de la accesibilidad, o la previsión de ulteriores y progresivas expansiones de la universidad, o la necesidad de servicios complementarios. Una sinergia que generada por "el impulso compartido por el puerto y la ciudad de los planes y proyectos concretos sobre el frente marítimo es la culminación de un proceso de acuerdo que casi siempre lleva a buenos resultados" (Alemany, 2005, 2). Y en cuarto lugar, los efectos de la operación urbanística universitaria sobre el contexto circundante no son fácilmente controlables y requieren "prudencia".

En la formalización de estas modalidades de cooperación, la existencia de una simbiosis dinámica entre las universidades y las ciudades medias es innegable. Incluso las ciudades con una base económica compleja, no pueden prescindir de los beneficios que significa la presencia de centros de enseñanza superior. Con este entorno, la universidad ha de desenvolver estrategias de actuación más allá de las funciones tradicionales de docencia e investigación. Es preciso que se implique en una tercera actividad que se sumaría a los dos cometidos enunciados. Una actividad de la institución académica que se concreta en las aportaciones directas sobre el bienestar social y el desarrollo económico de su entorno (MolasGallart, 2005). Por lo tanto, la Universidade da Coruña con su campus TIC podría garantizar la 


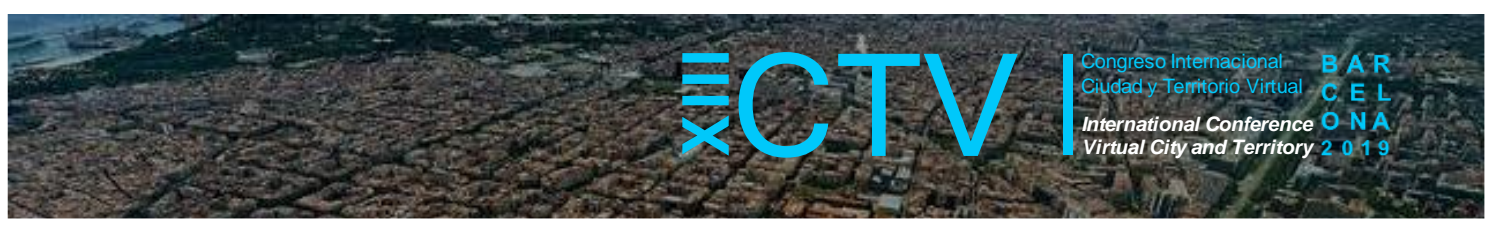

construcción de servicios y equipamientos colectivos para el uso de toda la ciudad que, en muchos casos, no responden a un uso específico ni extraordinario. Antes, al contrario, integran una serie equipamientos comunitarios que conllevan la recualificación urbana de un ámbito, con el espacio público como elemento de vertebración.

\section{Reflexiones finales}

Los parámetros aplicados al área portuaria de los muelles de Batería y Calvo Sotelo establecen las cualidades que confirman su adecuación como destino de actividades científicas de investigación y de enseñanza superior.

La primera de las cualidades hace referencia a la singularidad del enclave marítimo. Las sucesivas capas sedimentadas a lo largo del tiempo confieren al recinto portuario un carácter propio e identificable, que se potencia al introducir el uso universitario. La arquitectura del saber se cimienta, pues, en una premisa esencial: la concepción de la misma no es tanto la de un objeto, como la de un proceso, que construye un espacio común con el cual pueda identificarse la ciudadanía.

La segunda, la naturaleza reconocible del lugar, alude a los valores patrimoniales del tejido construido en el área portuaria, sean construcciones industriales, edificios en altura, o villas urbanas singulares. Elementos construidos que definen la imagen del lugar y cuya rehabilitación ha de concordar con el contenido funcional que albergarán, en un sentido utilitario, pero también representativo y simbólico.

La tercera de ellas, la potencialidad del espacio para acoger usos complementarios, sugiere la necesidad de una rigurosa planificación del ámbito portuario. Una recomposición encaminada a conferir un orden global a un espacio tan sectorizado como el puerto. Una estrategia que implemente el uso universitario en los muelles de Batería y Calvo Sotelo complementando tanto el tradicional pesquero, que se desenvuelve en los muelles de Linares Rivas y A Palloza, como el turístico, que tiene lugar en el muelle de Trasatlánticos.

Y la cuarta, la latente accesibilidad peatonal al mar en el área portuaria, adquiere un rango preferente en las relaciones de movilidad urbana. La posición central del recinto portuario, ocupando el plano bajo de la ciudad, propicia el uso de medios alternativos de movilidad personal. Favorece la utilización del área, tanto de los equipamientos como de los espacios públicos, por la juventud -el segmento de la población más dinámico- en horarios y con fines diversos sean académicos e investigadores, culturales, deportivos o de ocio informal.

En paralelo a estas cualidades, las actividades de transferencia del conocimiento y la tecnología constituyen un recurso relevante. Por ello, el territorio de la universidad no se circunscribe a los límites que marca el campus, sino que se define por las relaciones con los agentes territoriales y sociales. Una simbiosis que se convierte en motor de crecimiento económico y social. Conformada la zona como polo tecnológico, dichas actividades devienen instrumentos capaces de revitalizar el espacio urbano-portuario central de A Coruña.

De esta manera, al igual que la ciudad ha de completarse con un desarrollo medioambiental y socialmente sostenible, los muelles de Batería y Calvo Sotelo se reconocen como el lugar idóneo para ubicar el Campus TIC la UDC, una actividad que apunta al futuro. En ulteriores 


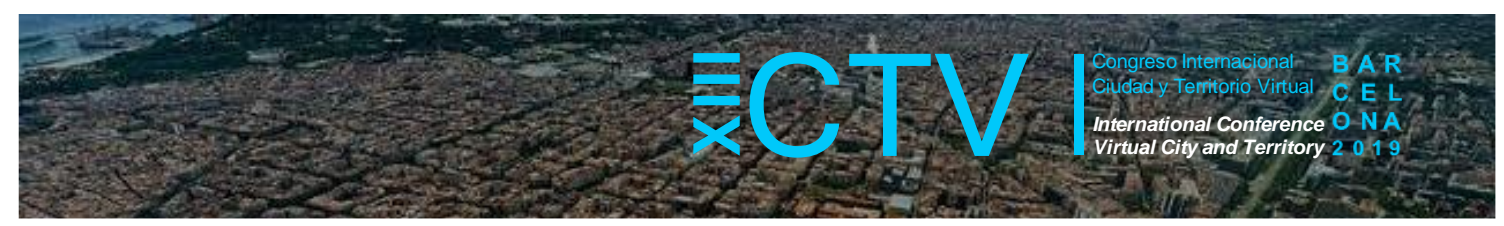

líneas de investigación, el proceso metodológico seguido puede ser reproducido para evaluar otras áreas portuarias que precisen ser regeneradas. En ellas, esos estudios deberían considerar la introducción de las actividades universitarias para reforzar la conexión entre puerto y ciudad.

Contribuciones de los autores: Los autores han trabajado de manera conjunta.

Conflicto de Intereses: Los autores declaran que no hay conflicto de intereses.

\section{Bibliografía}

Acinas García, J. R. (1998). El puerto es un proyecto permanente. Evolución del puerto-ciudad de La Coruña. A Coruña, España: Autoridad Portuaria de A Coruña.

Alemany, J. (2005). El frente marítimo, entre el urbanismo y la planificación portuaria. Portus, $10,2-3$.

Alemany, J. (2006). Rehabilitar, reconvertir, revitalizar, pero mantener el puerto en la ciudad. Neutra, 14, 22-25.

Autoridad Portuaria de A Coruña (coord.) (2018). Memoria Anual 2017. Puerto de A Coruña. A Coruña, España: Autoridad Portuaria de A Coruña. Recuperado de http://www.puertocoruna.com/es/autoridad-portuaria/actualidad/publicaciones.html

Barragán Muñoz, J. M. (1995). Puerto, ciudad y espacio litoral en la Bahía de Cádiz: las infraestructuras portuarias en la ordenación del espacio litoral de la Bahía de Cádiz. Cádiz, España: Autoridad Portuaria de la Bahía de Cádiz.

Boubacha, E., Davoult, D. \& Gueguen, E. (1997). Ville et Port. Mutation et Recomposition, note de synthèse et bibliographie. Paris-La Défense, Francia: Éd. Villes \& Territoires.

Camagni, R. (1998). La ciutat a Europa: globalització, cohesió i desenvolupament sostenible. Revista Econòmica de Catalunya, 36, 30-50.

Casariego, J., Guerra, E., Ley, P. y Palop, J. (1999). Waterfronts de nuevo. Transformaciones en los frentes urbanos de agua. Las Palmas de Gran Canaria, España: Ayuntamiento de Las Palmas de Gran Canaria.

Fernández Pérez, J. (1999). Frentes Marítimos en áreas portuarias. Puertos del Estado: Boletín Información Mensual del Ministerio de Fomento, 61, 11-15.

Grindlay Moreno, A. L. (2008). Ciudades y Puertos. Ciudades, 11, 53-80.

Hoyle, B. S. (1994). Development dynamics at the port-city interface. En B.S. Hoyle, D.A. Pinder \& M.S. Husain. (Eds.), Revitalising the waterfront: International dimension of dockland redevelopment (pp. 3-19). London, UK: Belhaven Press.

Hoyle, B. (1997). The new waterfront: principles, perceptions and practice in the UK and Canada. Mediterráneo, 10/11, 149-168.

Jacobs, J. (2011). Muerte y vida de las grandes ciudades. (Trad. Ángel Abad, del original The Death and Life of Great American Cities, 1961). Madrid, España: Capitán Swing Libros 


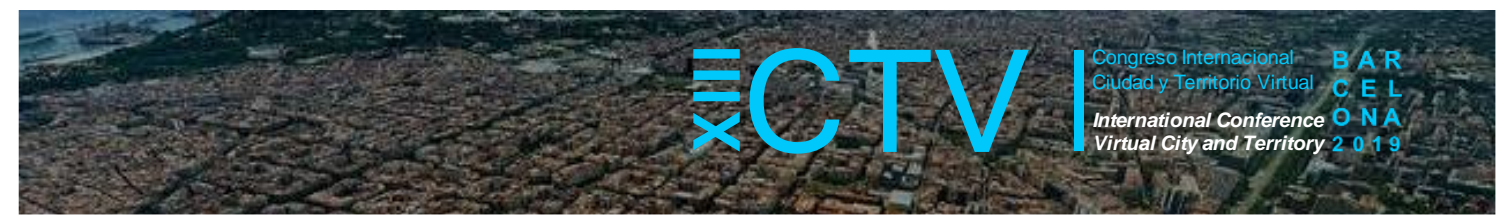

Martín, F. (2009). Puertos, ciudades y paisajes. Vivir y trabajar en ciudades portuarias. Portus, $18,56-61$.

Meyer, H. (1999). City and Port. Transformation of Port Cities. London, Barcelona, New York, Rotterdam. Rotterdam, Holanda: International Books.

Molas Gallart, J. (2005). Definir, quantificar i finançar la universitat. La tercera missió: un debat sobre el futur de la universitat. Coneixement $i$ Societat: Revista d'Universitats, Recerca $i$ Societat de la Informació, 5 (7), 6-27.

Muñoz, F. (2006). On the waterfronts. Ciudad sostenible, puerto consumible. Neutra, 14, 36-45.

Navarro Vera, J. R. (1998). Puerto y Ciudad en la Comunidad Valenciana. Alicante, España: Universidad de Alicante.

Navas, M. (2008). Guía para la elaboración de memorias de sostenibilidad en el Sistema Portuario Español. Valencia, España: Autoridad Portuaria de A Coruña, Autoridad Portuaria de Valencia y Organismo Público Puertos del Estado.

Peña, N. C. (2012). Reconversión de las áreas circundantes del puerto San Souci, su impacto urbano y socioeconómico. Santo Domingo, República Dominicana (Tesis del Máster en Gestión y Valoración Urbana). Universidad Politécnica de Cataluña, Barcelona.

Pozueta, J. (1996). Transformación de espacios portuarios en áreas urbanas. Experiencia española y marco internacional. Urbanismo COAM, 27, 7-17.

Reiriz Rey, J. M. (1999). La Coruña: hechos y figuras. A Coruña, España: Internós

Samperi, S. (1986). Getting it Started: The Public Sector. En A.R. Fitzgerald. (Ed.), Waterfront Planning and Development (pp. 47-53). New York, USA: American Society of Civil Engineers.

Soraluce Blond, J. R. (1985). Castillos y fortificaciones de Galicia. La arquitectura militar de los siglos XVI-XVII. La Coruña, España: Fundación Pedro Barrié de la Maza.

United Nations World Commission on Environment and Development (WCED) (1987). Nuestro futuro común. Informe sobre el Medio Ambiente y el Desarrollo [Informe Brundtland]. Recuperado de http://www.ecominga.uqam.ca/PDF/BIBLIOGRAPHIE/GUIDE LECTURE 1/CM MAD-Informe-Comision-Brundtland-sobre-Medio-Ambiente-Desarrollo.pdf

Uriarte, I. (2007). Plazas del Agua. Metamorfosis del espacio portuario en las villas vascas. Portus, 14, 54-57.

Uribe, C. de (1886). Reseña de los proyectos y de las obras del puerto de La Coruña: Muelles embarcaderos. Revista de Obras Públicas, XIV, 177-180.

Vigo Trasancos, A. (2014). A Coruña. Historia e imagen de un puerto atlántico (s. I-1936). En Mà . del Mar Lozano Bartolozzi, y V. Méndez Hernán. (Coords.), Patrimonio cultural vinculado con el agua. Paisaje, urbanismo, arte, ingeniería y turismo (pp. 381-394). Mérida (Cáceres), España: Editora Regional de Extremadura, Universidad de Extremadura y Gobierno de Extremadura.

Vila y Algorri, E. (1909). Memoria Histórica y Descriptiva de las obras del puerto de La Coruña. La Coruña, España: Imprenta y Fotograbado de Ferrer. 